\title{
FINITE GROUPS IN WHICH THE DEGREES OF THE NONLINEAR IRREDUCIBLE CHARACTERS ARE DISTINCT
}

\author{
YAKOV BERKOVICH, DAVID CHILLAG, AND MARCEL HERZOG
}

(Communicated by Warren J. Wong)

Dedicated to I. Martin Isaacs on the occasion of his sixtieth birthday

\begin{abstract}
Finite groups in which all the nonlinear irreducible characters have equal degrees were described by Isaacs, Passman, and others. The purpose of this article is to consider the other extreme, namely, to characterize all finite groups in which all the nonlinear irreducible characters have distinct degrees.
\end{abstract}

Finite groups in which all the nonlinear irreducible characters have equal degrees were described by Isaacs, Passman, and others (see [8, Chapter 12]). The purpose of this article is to consider the other extreme, namely, the case when all nonlinear irreducible character degrees are distinct. We prove

Theorem. Let $G$ be a nonabelian finite group. Let $\left\{\theta_{1}, \theta_{2}, \ldots, \theta_{r}\right\}$ be the set of all nonlinear irreducible ordinary characters of $G$. Assume that $\theta_{i}(1) \neq \theta_{j}(1)$ for all $i \neq j$. Then one of the following holds:

(1) $G$ is an extra-special 2-group.

(2) $G$ is a Frobenius group of order $p^{n}\left(p^{n}-1\right)$ for some prime power $p^{n}$ with an abelian Frobenius kernel of order $p^{n}$ and a cyclic Frobenius complement.

(3) $G$ is a Frobenius group of order 72 in which the Frobenius complement is isomorphic to the quaternion group of order 8 .

Remarks. All the groups described above satisfy the assumption of the theorem. The groups of type (1) and (2) have exactly one nonlinear character degree, while the group in (3) has two such character degrees. To show that there are no perfect groups satisfying the assumption, we use the classification of the finite simple groups. The proof for nonperfect groups is independent of the classification.

In [10], Seitz shows that if in the theorem $r=1$, then either (1) or (2) holds.

Notation. Most of our notation is standard and taken mainly from [8]. We will denote the set of all irreducible ordinary characters of the finite group $G$ by

Received by the editors September 7, 1990, and, in revised form, February 1, 1991.

1980 Mathematics Subject Classification (1985 Revision). Primary 20CXX.

This paper was written during the summer of 1990, when the second and third authors were visiting the University of Mainz, a visit supported by the DFG as part of the Darstellungstheorie project. We would like to thank the DFG for its support and the University of Mainz for its hospitality. 
$\operatorname{Irr}(G)$. For the purpose of this paper we call a finite nonabelian group a $D$ group if it satisfies the assumption of the theorem, namely, for any two distinct nonlinear $\theta, \eta \in \operatorname{Irr}(G)$, we have that $\theta(1) \neq \eta(1)$. A final piece of notation is the following:

Let $G$ be a finite group and $H$ a proper nontrivial normal subgroup of $G$. We say that $(G, H)$ is a Camina pair if $\left|C_{G}(x)\right|=\left|C_{G / H}(x H)\right|$ for every $x \in G-H$. Camina showed [1] that if $(G, H)$ is a Camina pair and $G$ is not a Frobenius group with the Frobenius kernel $H$, then either $G / H$ or $H$ is a $p$-group for some prime $p$.

We prove the theorem via a series of lemmas.

Lemma 1. Let $G$ be a D-group. Then

(1) $G / N$ is a D-group or abelian for every normal subgroup $N$ of $G$.

(2) If $\chi$ is a nonlinear irreducible character of $G$, then $\chi$ is rational-valued.

(3) $G$ is not a perfect group; that is, $G^{\prime}<G$.

(4) $\left(G, G^{\prime}\right)$ is a Camina pair.

(5) $Z(G) \subseteq G^{\prime}$.

Proof. (1) This is clear because $\operatorname{Irr}(G / N) \subseteq \operatorname{Irr}(G)$.

(2) Let $\epsilon$ be a $|G|$ th root of unity and $\sigma \in \operatorname{Gal}(Q(\epsilon) / Q)$. Then $\chi^{\sigma} \in \operatorname{Irr}(G)$ and $\chi^{\sigma}(1)=\chi(1)$. As $G$ is a $D$-group, $\chi^{\sigma}=\chi$, so $\chi^{\sigma}(x)=(\chi(x))^{\sigma}=\chi(x)$ for all $x \in G$. Hence, $\chi(x)$ is rational for all $x \in G$.

(3) Suppose that $G=G^{\prime}$. Let $N$ be a normal subgroup of $G$ such that $G / N$ is a composition factor. By (1) we get that $G / N$ is a nonabelian simple $D$-group and by (2) that if $\theta \in \operatorname{Irr}(G / N)-\left\{1_{G / N}\right\}$, then $\theta$ is rational-valued. By a theorem of Feit and Seitz ([6, Corollary B1]), $G / N \cong \mathrm{Sp}_{6}(2)$ or $O_{8}^{+}(2)$. Examining [4], we see that neither $\operatorname{Sp}_{6}(2)$ nor $O_{8}^{+}(2)$ is a $D$-group. This contradiction proves that $G^{\prime}<G$.

(4), (5) Let $\chi$ be a nonlinear irreducible character of $G$ and $\lambda$ a linear character of $G$. Then $\lambda \chi \in \operatorname{Irr}(G)$ and $(\lambda \chi)(1)=\chi(1)$. Since $G$ is a $D$-group, $\lambda \chi=\chi$. So $\lambda(x) \chi(x)=\chi(x)$ for all $x \in G$. Hence $\chi(x)=0$ for all $x$ 's such that $\lambda(x) \neq 1$. Thus $\chi$ vanishes off the subgroup $\operatorname{Ker}(\lambda)$. Since this is true for all linear $\lambda \in \operatorname{Irr}(G)$, we conclude that $\chi$ vanishes off

$$
\bigcap\{\operatorname{ker}(\lambda) \mid \lambda \in \operatorname{Irr}(G), \lambda(1)=1\}=G^{\prime} .
$$

We further conclude that each nonlinear character of $G$ vanishes off $G^{\prime}$.

Now let $g \in G-G^{\prime}$. As $G / G^{\prime}$ is abelian, we have that $C_{G / G^{\prime}}\left(g G^{\prime}\right)=G / G^{\prime}$. On the other hand, $\chi(g)=0$ for all nonlinear $\chi \in \operatorname{Irr}(G)$, and consequently

$$
\begin{aligned}
\left|C_{G}(g)\right| & =\sum\left\{|\lambda(g)|^{2} \mid \lambda \in \operatorname{Irr}(G), \lambda(1)=1\right\} \\
& =|\{\lambda \in \operatorname{Irr}(G), \lambda(1)=1\}|=\left|G: G^{\prime}\right| .
\end{aligned}
$$

Thus, $\left|C_{G / G^{\prime}}\left(g G^{\prime}\right)\right|=\left|C_{G}(g)\right|$, and $\left(G, G^{\prime}\right)$ is a Camina pair. This proves (4).

Let $z \in Z(G)$, and let $\chi$ be a nonlinear irreducible character of $G$. Then $\chi(z) \neq 0$ and, since $\chi$ vanishes off $G^{\prime}$, we get that $z \in G^{\prime}$. This proves (5).

Lemma 2. Let $G$ be a p-group for some prime $p$. If $G$ is a D-group, then $G$ is a 2-group in which $\left|G^{\prime}\right|=|Z(G)|=2$. That is, $G$ is an extra-special 2-group.

Proof. By Lemma $1, G$ has a real-valued nonprincipal irreducible character, so that $|G|$ is even $\left[8,(3.16)\right.$, p. 46]. It follows that $p=2$. Write $|G|=2^{m}$, 
and let $\mathscr{A}=\left\{\theta_{1}, \theta_{2}, \ldots, \theta_{s}\right\}$ be the set of all nonlinear irreducible ordinary characters of $G$. Let $\theta \in \mathscr{A}$ be of maximal degree. Set $\theta(1)=2^{r}$. As $G$ is a $D$-group, we have that $\left\{\theta_{1}(1), \theta_{2}(1), \ldots, \theta_{s}(1)\right\} \subseteq\left\{2,2^{2}, 2^{3}, \ldots, 2^{r}\right\}$. Now,

$$
|G|=\left|G: G^{\prime}\right|+\sum_{i=1}^{s}\left(\theta_{i}(1)\right)^{2} \leq\left|G: G^{\prime}\right|+\sum_{i=1}^{r} 2^{2 i}=\left|G: G^{\prime}\right|+2^{2} \frac{2^{2 r}-1}{2^{2}-1} .
$$

Observe that $(\theta(1))^{2}=2^{2 r}$ divides $|G|$, and therefore $2^{2 r} \leq|G| / 2$. Using this fact in $(*)$ we get that

$$
|G| \leq\left|G: G^{\prime}\right|+2^{2} \frac{|G|-2}{\left(2^{2}-1\right) 2}=\left|G: G^{\prime}\right|+\frac{2}{3}|G|-\frac{4}{3} .
$$

Suppose that $\left|G^{\prime}\right| \geq 4$. Then $|G| \leq \frac{1}{4}|G|+\frac{2}{3}|G|-\frac{4}{3}$, which means that $\frac{1}{12}|G| \leq$ $-\frac{4}{3}$. Since this is impossible, we get that $\left|G^{\prime}\right|=2$. By Lemma 1(5), we know that $Z(G) \subseteq G^{\prime}$ and consequently $|Z(G)|=2$. So $G$ is an extra-special 2group.

In the next two lemmas we use the known information on the irreducible characters of Frobenius groups; see for example [5, Theorem 13.8 and its proof].

Lemma 3. Let $G$ be a D-group which is a Frobenius group with an abelian Frobenius kernel $K$. Then $|G|=|K|(|K|-1)$, and $|K|=p^{m}$ for some prime $p$ and a natural number $m$.

Proof. Let $H$ be a Frobenius complement and $\theta \in \operatorname{Irr}(K)-\left\{1_{K}\right\}$. Then $\theta^{G} \in$ $\operatorname{Irr}(G)$ and $\theta^{G}(1)=|G: K|$. As $G$ is a $D$-group, $G$ has exactly one irreducible character of degree $|G: K|$. This means that all the $|K|-1$ elements of $\operatorname{Irr}(K)-$ $\left\{1_{K}\right\}$ comprise one orbit under the action of $H$. But $H$ acts with no fixed points on $\operatorname{Irr}(K)-\left\{1_{K}\right\}$; thus, $|H|=|K|-1$.

Let $p$ be a prime divisor of $|K|$ and $P$ a Sylow $p$-subgroup of $K$. Write $K=P \times R$, with $R$ a $p$-complement. Then $G / R$ is a Frobenius group with Frobenius kernel isomorphic to $P$ and Frobenius complement isomorphic to $H$. As $G / R$ is a $D$-group, the argument in the first paragraph shows that $|H|=|P|-1$ and hence $K=P$. (The fact that $|K|$ is a prime power follows also from the known fact that the degree of a doubly transitive Frobenius group is a prime power.)

Lemma 4. Let $G$ be a D-group which is a Frobenius group with the Frobenius kernel $K$. Then $K$ is abelian.

Proof. Let $H$ be a Frobenius complement. Assume that $K$ is not abelian. We seek a contradiction.

Without loss of generality we may assume that $[K, K, K]=1$. Then $K$ is nilpotent of class 2; in particular, $K^{\prime} \leq Z(K)$. Let $X$ be either $K^{\prime}$ or $Z(K)$. Then $G / X$ is a $D$-group, which is a Frobenius group with abelian Frobenius kernel $K / X$ and a Frobenius complement isomorphic to $H$. It follows from Lemma 3 that $|H|=|K / X|-1$. Thus $\left|K / K^{\prime}\right|=|K / Z(K)|$ so that $K^{\prime}=Z(K)$.

Let $\mathscr{L}_{1}, \mathscr{L}_{2}, \ldots, \mathscr{L}_{r}$ be the orbits of the fixed-point-free action of $H$ on $\operatorname{Irr}(K)-\left\{1_{K}\right\}$. We may assume that $\mathscr{L}_{1}$ consists of linear characters. Then $\left|\mathscr{L}_{i}\right|=|H|=\left|K / K^{\prime}\right|-1$ for each $i$. Let $\theta \in \mathscr{L}_{i}$ and $\eta \in \mathscr{L}_{j}$ for some $i \neq j$. Then $\theta^{G} \neq \eta^{G}$ and $\theta^{G}, \eta^{G}$ are two nonlinear irreducible characters of $G$. As $G$ is a $D$-group, $\theta^{G}(1) \neq \eta^{G}(1)$, which implies that $\theta(1) \neq \eta(1)$. It follows that different orbits contain characters of different degrees. 
Let $\chi$ be a nonlinear irreducible character of $K$. Set $\chi_{Z(K)}=e \lambda$ with $\lambda \in \operatorname{Irr}(Z(K))$ and $e$ a positive integer. We claim that $\left(\theta_{Z(K)}, \lambda\right)=0$ for all $\theta \in \operatorname{Irr}(K)-\{\chi\}$. For if $\left(\theta_{Z(K)}, \lambda\right) \neq 0$ for some $\theta \in \operatorname{Irr}(K)-\{\chi\}$, then both $\theta$ and $\chi$ are constituents of $\lambda^{K}$. As $K / Z(K)$ is abelian, we get that $\chi=\alpha \theta$, where $\alpha \in \operatorname{Irr}(K / Z(K))$ (see [8,6.2, p. 95]). As $\alpha$ is linear, $\chi(1)=\theta(1)$, and hence $\{\chi, \theta\} \subseteq \mathscr{L}_{i}$ for some $i>1$. It follows that $\chi=\theta^{x}$ for some $x \in H-\{1\}$. Next, $\theta_{Z(K)}=f \lambda$ with $f$ a positive integer, and therefore $f \lambda^{x}=e \lambda$ so that $\lambda=\lambda^{x}$. Since $Z(K) H$ is a Frobenius group with $Z(K)$ the Frobenius kernel and $H$ a complement, we get that $\lambda=1_{Z(K)}$. But then $Z(K) \subseteq \operatorname{Ker}(\chi)$ and $\chi \in \operatorname{Irr}(K / Z(K))$, forcing $\chi$ to be linear, a contradiction. Thus no such $\theta$ exists. It follows that $\lambda^{K}=e \chi$, and so $\chi$ vanishes on $K-K^{\prime}=K-Z(K)$.

Take $x \in K-Z(K)$; then $\chi(x)=0$ for all nonlinear $\chi \in \operatorname{Irr}(K)$, and so $\left|C_{K}(x)\right|=1+\left|\mathscr{L}_{1}\right|=\left|K: K^{\prime}\right|=|H|+1$. Note that $Z(K)<\langle Z(K), x\rangle \leq C_{K}(x)$, and therefore $|Z(K)|<\left|C_{K}(x)\right|=|H|+1$. On the other hand $Z(K) H$ is a Frobenius group with Frobenius kernel $Z(K)$ and Frobenius complement $H$. This implies that $|H|$ divides $|Z(K)|-1$ so that $|H|+1 \leq|Z(K)|$. This contradiction completes the proof.

Proof of the theorem. By Lemma $1,\left(G, G^{\prime}\right)$ is a Camina pair. If $G$ is a $p$ group, then by Lemma 2, $G$ satisfies (1) of the theorem. If $G$ is a Frobenius group with Frobenius kernel $G^{\prime}$, then Lemma 4 implies that $G^{\prime}$ is abelian, and by Lemma 3 we get that $|G|=\left|G^{\prime}\right|\left(\left|G^{\prime}\right|-1\right)$ with $\left|G^{\prime}\right|$ a prime power. Also a Frobenius complement isomorphic to $G / G^{\prime}$ is abelian and hence cyclic. Then $G$ satisifes (2) of the theorem (see [7, p. 508]). Thus we may assume that $G$ is neither a $p$-group nor a Frobenius group with $G^{\prime}$ the Frobenius kernel. By [1], either $G^{\prime}$ or $G / G^{\prime}$ is a $p$-group for some prime $p$. Camina pairs like that are called $F 2(p)$ groups in [2] and [3].

If $G^{\prime}$ is a $p$-group, then $O_{p^{\prime}}\left(G / G^{\prime}\right)=1$ by [2, Lemma 4.4]. But $G / G^{\prime}$ is abelian, and hence $G / G^{\prime}$ is a $p$-group, forcing $G$ to be a $p$-group, a contradiction. Thus $G / G^{\prime}$ is a $p$-group. Let $P$ be a Sylow $p$-subgroup of $G$. By [2, Corollary 3.5], $P$ is not abelian. By [3, Lemma 2.1], $G$ has a normal $p$-complement, say $R$. Then $G=R P$, with $R \cap P=1$. By Lemma 1 , $G / R \cong P$ is a $D$-group, and now Lemma 2 implies that $P$ is a 2 -group with $\left|P^{\prime}\right|=|Z(P)|=2$. In particular, the nilpotency class of $P$ is 2 . [2, Theorem 5.1] now implies that $G$ is a Frobenius group with Frobenius complement isomorphic to $Q_{8}$, the quaternion group of order 8 . Finally, Lemmas 4 and 3 imply that the Frobenius kernel has order 9, so $G$ satisfies (3) of the theorem.

\section{ACKNOWLEDGMENT}

We wish to thank the referee for his suggestions and very helpful remarks.

\section{REFERENCES}

1. A. Camina, Some conditions which almost characterize Frobenius groups, Israel J. Math. 31 (1978), 153-160.

2. D. Chillag and I. D. Macdonald, Generalized Frobenius groups, Israel J. Math. 47 (1984), 111-123.

3. D. Chillag, A. Mann, and C. Scoppola, Generalized Frobenius groups. II, Israel J. Math. 62 (1988), 269-282. 
4. J. H. Conway, R. T. Curtis, S. P. Norton, R. A. Parker, and R. A. Wilson, Atlas of finite groups, Oxford Univ. Press (Clarendon), Oxford and New York, 1985.

5. L. Dornhoff, Group representation theory, Part A, Marcel Dekker, New York, 1971.

6. W. Feit and G. M. Seitz, On finite rational groups and related topics, Illinois J. Math. 33 (1988), 103-131.

7. B. Huppert, Endliche Gruppen. I, Springer-Verlag, Berlin, Heidelberg, and New York, 1967.

8. I. M. Isaacs, Character theory of finite groups, Academic Press, New York, 1976.

9. D. S. Passman, Permutation groups, Benjamin, New York and Amsterdam, 1968.

10. G. M. Seitz, Finite groups having only one irreducible representation of degree greater than one, Proc. Amer. Math. Soc. 19 (1968), 459-461.

Department of Mathematics, Bar-Ilan University, Ramat Gan 52900, IsRael

Department of Mathematics, Technion, IsRael Institute of Technology, Haifa 32000, ISRAEL

School of Mathematical Sciences, Raymond and Beverly Sackler faculty of Exact Sciences, Tel-Aviv University, Tel-Aviv, IsRael 